\title{
A qualitative study exploring perceptions of policymakers about community pharmacy practice and extended pharmacy services in Lahore, Pakistan
}

Background: Evidence-based policymaking is critical for improving the standards of pharmacy practice. Within this context, the present study aimed to explore the perception of health policymakers (PMs) about the standards of community pharmacy practice in Lahore, Pakistan.

Methodology: A qualitative approach was used to gain an in-depth knowledge of PMs about the community pharmacy practice and EPS in Lahore. Government officials belonging to the Drug Authority of Pakistan and involved in regulation and policy-making were targeted for the study. Seven respondents were interviewed through a validated semi-structured interview guide. All interviews were audio-taped, transcribed verbatim, and were then analyzed for thematic content analysis.

Results: Thematic content analysis yielded four major themes, (1) Lack of law enforcement (2) Support for EPS (3) Need for the change in the pharmacy system (4) Barriers toward practice change. Though PMs encouraged the implementation of EPS, their understanding of the true concept of EPS was questionable. Several barriers towards practice change were identified by the PMs.

Conclusion: Pharmacists' active involvement in community practice, enforcement of legislation, and revisiting pharmacy curriculum could lay the foundations for the standardisation of community pharmacy practice in Lahore, Pakistan. 


\section{Introduction}

The profession of pharmacy has evolved globally in the past few decades. However, community pharmacy practice in Pakistan still needs a paradigm shift to improve the standards of pharmacy practice. ${ }^{[1]}$ The necessary changes can be brought in through evidence-based policymaking. Several countries have successfully implemented the policies to equip community pharmacists with patient-centred responsibilities. ${ }^{[2]}$

Given the importance of policymaking in improving the standards of pharmacy practice, there is a paucity of data in this area from Pakistan. The objective of this study was to explore the perceptions of policymakers about the current standards of community pharmacy practice in Pakistan, practice change, and extended pharmacy services (EPS) in Pakistan.

\section{Methods}

A qualitative methodology was adopted to explore the perception of PMs regarding community pharmacy settings in Lahore, Pakistan. Based on an in-depth literature review and current prevailing practices of community pharmacy in Pakistan, a semi-structured interview guide was developed. ${ }^{[3]}$ The guide focused on knowledge of EPS, perception towards current community pharmacy practice in Pakistan, knowledge, and confidence in practice change, customers' feedback and future interventions towards practice change. The guide was subjected to validation and reliability assessment prior to data collection. Qualitative experts at Universiti Sains Malaysia validated the interview guide by using a combination of argumentative and cumulative techniques. ${ }^{[4]}$ The guide was piloted on two policymakers and was modified accordingly. Reliability was assured by preserving scrupulous records of the 
interviews. As mutual consent of the experts and PMs was received, the interview guide was made available for real-time study.

Participants were recruited using purposive sampling method until saturation was achieved. In-depth interviews with consented participants were conducted after individual appointments. The interviews were conducted in English and each interview lasted from 30 to 40 minutes. Probing questions were asked and participants were given freedom to express additional views and comments. All interviews were audio-recorded and the principal researcher took additional field notes. The saturation was achieved at the $5^{\text {th }}$ interview; however, two interviews were carried out further to confirm the saturation. The interviews were verified for accuracy and consistency by listening to the recordings. The first author analyzed the transcripts line by line, which was read repeatedly. The transcripts were analysed for identification of themes using thematic content analysis (TCA) technique. The identified themes were reviewed by another researcher independently in context of the transcripts. After a series of discussions between the two researchers, a consensus was reached about the themes that should be included in the subsequent analysis. Research question and study objectives guided the selection of themes throughout the process Figure 1.

The study was approved by Humans Ethics Committee (HEC), University College of Pharmacy, University of the Punjab, Lahore, Pakistan (D/HEC/100/UCP1923).

\section{Results}

Participants' characteristics are presented in table 1. The analysis of seven in-depth interviews yielded four themes. 


\section{Theme 1: Lack of law enforcement}

Majority of the PMs did not show confidence in the current status of the community pharmacy practice in the province of Punjab. Respondents believed that the laws to regulate the profession of pharmacy exist but barriers to improvement in the current standards in the lack of law enforcement.

\section{"I am not satisfied at all because the government has done a lot but the implementation and enforcement is not there" (PM2)}

\section{Theme 2: Support for EPS}

Respondents identified the importance of EPS from patients' perspectives and consider it an important part of pharmacist responsibilities.

"Definitely EPS would ensure the provision of quality medicines and better health care to the patients" (PM4)

However, it was also observed that the participants were not clear in their thoughts about the type of services regarded as EPS.

"Well it is pharmacist's duty to counsel the patients at the time of dispensing drug" (PM3)

\section{Theme 3: Need for the change in the pharmacy system}

Participants agreed that there is a need to change the basic structure of community pharmacy settings in Pakistan. However, they believed that the involvement of pharmacists in community settings is essential to bring this change.

"There is a dire need to change the practice I think the real future of pharmacist is in community pharmacy" (PM7) 
"Radical change is required in fact the whole system needs to be changed majority of the cases there are no community pharmacy services rather product based services" (PM3)

\section{Theme 4: Barriers toward practice change}

Pharmacists' attitudes, Pharmacy Act 1967, and the role of academic were regarded as the major barriers towards practice change.

"The First barrier is academia as they don't have practical exposure, second is regulation and third the inter-professional recognition" (PM6)

"The current Pharmacy Act 1967 is the biggest barrier, amendment in this act is necessary" (PM1)

"Well, in my opinion, the biggest barrier is the economy of the country and the confidence and mind-set of the fresh pharmacists" (PM4)

\section{Discussion}

Lack of law enforcement remains the major issue in current pharmacy settings in Lahore, Pakistan. The Government of Punjab amended the rules for the regulation of pharmacies and sales of drugs in the province of Punjab, known as Punjab Drug Rules 2007. ${ }^{[5]}$ These rules aimed to ensure the sales and dispensing of drugs under direct the supervision of qualified pharmacist with round the clock presence in community pharmacies all around the province of Punjab. However, many pharmacies in Punjab are still operating without pharmacists. 
Strict measures need to be taken from the stakeholders to ensure the enforcement of the law as it is critical for the betterment of pharmacy practice in Lahore, Punjab.

Pharmacists in Pakistan are still underutilized and their role as health care professionals is not yet recognized either by the community or other healthcare providers. ${ }^{[6]}$ Lack of interests of Pakistani pharmacists towards community pharmacy has also contributed to their low recognition. ${ }^{[7]}$ Dominance of non-pharmacists in pharmacy retail industry and negative prospects of financial remuneration could possibly explain the current situation. ${ }^{[8]}$ It would be safe to say that policy change alone may not help the cause of improving pharmacy practice in Lahore as academia needs to play an equally important role to emphasise the importance of community pharmacist in health settings during the 5-year undergraduate program. Model pharmacies need to be established and regular visits to those pharmacies should be arranged to enhance students' interests in taking on the role community pharmacists.

\section{Conclusions}

Lack of law enforcement has significantly hampered the evolvement of pharmacy practice in Lahore, Pakistan. There is a dire need for multidimensional efforts from policymakers, pharmacists, academia to bring a much-needed change in the community pharmacy practice in Pakistan.

\section{Reference}

1. Khowaja K. Healthcare systems and care delivery in Pakistan. J Nurs Admin 2009; 39(6): 263-265.

2. Mossialos E, Naci H, Courtin E. Expanding the role of community pharmacists: Policymaking in the absence of policy-relevant evidence? Health Policy 2013; 111(2): 135-148. 
3. Olsson E, Ingman P, Ahmed B, Sporrong SK. Pharmacist-patient communication in Swedish community pharmacies. Research in Social and Administrative Pharmacy 2014; 10(1): 149-155.

4. Conwell Y, Forbes NT, Cox C, Caine ED. Validation of a measure of physical illness burden at autopsy: the Cumulative Illness Rating Scale. Journal of the American Geriatrics Society 1993;41(1): 38-41.

5. Government of the Punjab. Punjab Drug Rules, 2007 Retrieved 4/7/2016, from http://www.pcdapakistan.com/wp-content/uploads/2013/04/PUNJAB-DRUGSRULES-2007.pdf

6. Azhar S, Hassali MA, Ibrahim MI, Ahmad M, Masood I, Shafie AA. The role of pharmacists in developing countries: the current scenario in Pakistan. Hum Resour Health 2009; 7: 54.

7. Sahir A, Sahir J, Shah MF. Archives of pharmacy practice on pharmacy education. Archives of Pharmacy Practice 2014;5(2): 95.

8. Aslam N, Bushra R, Khan MU. Community pharmacy practice in Pakistan. Archives of pharmacy practice 2012;3(4): 297. 